\title{
LiBeB Production and Associated Astrophysical Sites
}

\author{
Elisabeth Vangioni-Flam \\ Institut d'Astrophysique de Paris, 98 bis Bd Arago, 75014 Paris, France \\ Michel Cassé \\ Service d'Astrophysique, Orme des Merisiers, CEA, \\ 91191 Gif sur Yvette, France
}

\begin{abstract}
The various modes of spallative $\mathrm{LiBeB}$ production are summarized, and classified according to their dependence or independence on the abundance of medium heavy elements (CNO) illustrated by that of oxygen in the interstellar medium. The predictions of the models are confronted to the available observational correlations ( $\mathrm{Be}, \mathrm{B}$ vs $\mathrm{O}$ ). Clearly, a primary mechanism should lead to a slope one in the $\lg (\mathrm{Be} / \mathrm{H})$ vs $[\mathrm{O} / \mathrm{H}]$ plot and a secondary mechanism to a slope two. Due to the ambiguity of the $\mathrm{O}$ data, another criterion, based on energetics, can help us to select an adequate model. A purely secondary origin in the very early Galaxy is much more energy demanding than a primary one. Indeed, magnesium seems to be a possible surrogate of oxygen and iron since i) it is spectroscopically more easy to cope with and ii) its nucleosynthetic yield is independent of the mass cut and does not depend on metallicity.
\end{abstract}

\section{Introduction}

A turning point in the theory of the origin and evolution of light elements has been the observation of a linear relationship between both $\mathrm{Be}$ and $\mathrm{B}$ and $\mathrm{Fe}$ in metal poor halo stars (Rebolo, Molaro \& Beckman 1988; Duncan et al 1992, 1997; Gilmore et al 1992, Boesgaard \& King 1993; Ryan, Norris, Bessel \& Deliyannis 1994, Primas et al 1999). But recently, the debate has taken a complex turn, due to a modification of the O-Fe correlation indicated by the data of Israelian et al (1998) and Boesgaard et al (1999). This revision is however not universally admitted, and the debate is still open (e.g. Fulbright \& Kraft 1999; Gustafsson 1999; Reetz 2000). Since the last International Cosmic Ray Conference (Barring 2000) and the most recent LiBeB meeting (Ramaty et al 1999), the situation has not ceased to evolve.

Prior to the proposal of change of the $\mathrm{O}-\mathrm{Fe}$ relation, the situation was the following: in order to explain the observed proportionality of the $\mathrm{Be} / \mathrm{H}$ ratio to $\mathrm{Fe} / \mathrm{H}$ (itself taken proportional to $\mathrm{O} / \mathrm{H}$ in halo stars as implied at that time by the data and by the SNII calculated yields), it was necessary to invoke a primary production mechanism (i.e. a production rate of $\mathrm{LiBeB}$ independent of the interstellar metallicity), driven by the break up in flight of $\mathrm{C}$ and $\mathrm{O}$ colliding with $\mathrm{H}$ and $\mathrm{He}$ in the ISM (for reviews see Vangioni-Flam et al 1999b, 2000a,b). 
Now the reality of this primary component is questioned on the basis of the "new" O-Fe correlation. Concerning oxygen, however, the situation is not settled. The abundances derived from the forbidden (OI) line, which is certainly the most accurate source when it is not too weak, suggest a plateau but measurements of near IR OH band in dwarfs and subgiants lead to a rising trend with decreasing $\mathrm{Fe} / \mathrm{H}$. In contrast, Fulbright \& Kraft (1999) have analysed in great details the (OI) spectral region in the two metal poorest stars of the Israelian et al sample and have found a lower $\mathrm{O} / \mathrm{Fe}$ ratio. As stressed for instance by Pagel (1999), both methods, indeed, have their drawbacks and technical difficulties: the $\mathrm{OH}$ bands are subject to uncertainties in UV continuum absorption (Balachandran \& Bell 1998) and effective temperature, while the forbidden lines are so weak at low metallicity that the determination of the continuum becomes problematic.

So the situation is wide open and one is inclined to propose a different metallicity index, less ambiguous than $\mathrm{O}$. The closer element to $\mathrm{O}$ whose abundance is widely measured is $\mathrm{Mg}$. It has been chosen, due to its various advantages, both observational and theoretical, as the reference element to follow galactic evolution by Thomas et al (1998), Fuhrmann (1998) and Shigeyama \& Tsujimoto (1998). Indeed i) $\mathrm{Mg}$ seems easier to measure than $\mathrm{O}$ and ii ) $\mathrm{Mg}$ and $\mathrm{O}$ are coproduced in SNII explosions, which are the main sources of these elements (Woosley \& Weaver 1995, Thielemann, Nomoto \& Hashimoto, 1996). Moreover the $\mathrm{Mg}$ yield is independent of the mass cut, and does not significantly depend on metallicity (Umeda et al 2000). In the light of existing data, the $\mathrm{Mg} / \mathrm{Fe}$ vs $\mathrm{Fe} / \mathrm{H}$ correlation is rather flat up to $\mathrm{Fe} / \mathrm{H}=-1$, like that of other alpha elements (Mc Williams 1997, Pagel \& Tautvaisiene, 1995). Taken at face value, (assuming $\mathrm{Mg}$ proportional to $\mathrm{O}$, on nucleosynthetic grounds, neglecting the peculiar behaviour of the most massive stars which are marginal in the chemical evolution budget), these data indicate the need of a primary component, then we are back to the previous situation (Cassé et al 1995; Vangioni-Flam et al 1996, 1998; Ramaty et al 1996). Thus, on this sole basis, a purely secondary origin of $\mathrm{Be}$ in the halo (Fields \& Olive 1999a) driven by the standard GCR seems inadequate. However, the situation is not completely settled.

The outline of this paper is the following: in section 2 we recall the basic production mechanisms of $\mathrm{LiBeB}$, in section 3 we decline the astrophysical agents and sites, in section 4 , we compare the various models designed, in section 5 we propose key observations to remove the present ambiguities.

\section{Nucleosynthesis of LiBeB}

\subsection{Thermal production and destruction}

Thermal nucleosynthesis in the Big Bang produces negligible amounts of Be and B. Only ${ }^{7} \mathrm{Li}$ is synthesized in significant amounts. Moreover, $\mathrm{LiBeB}$ do not survive stellar temperatures except in a thin surface layer where they are observed, reflecting the interstellar composition inherited by the star at birth. ${ }^{7} \mathrm{Li}$ is however thought to be produced by AGB stars (Abia et al 1993) and novae (Hernanz et al 1996) and also by SNII through neutrino spallation of carbon. This neutrino spallation in carbon shells of type II supernovae is expected to produce 
also ${ }^{11} \mathrm{~B}$, but the yields are sensitive to the assumed temperature (energy) of the neutrinos, which is uncertain (see Hartmann et al 1999 for a review).

\subsection{Non Thermal production}

Nuclear spallation, i.e. the break up of medium heavy elements by collisions with protons and alphas remains the leading production process of light elements in the cosmos (Meneguzzi, Audouze \& Reeves 1971). In principle, all isotopes of interest are generated either by the interaction of fast $\mathrm{p}$ and alphas on CNO at rest in the ISM, or conversely by the interaction of fast $\mathrm{C}$ and $\mathrm{O}$ (principally) with ambient $\mathrm{H}$ and $\mathrm{He}$, supplemented by the alpha +alpha reaction giving rise exclusively to Li isotopes. (e.g. Reeves 1994).

The cross sections are well measured (Read \& Viola 1985; Webber et al 1990 a, b), and have been updated recently by Ramaty et al (1997). The hierarchy of the cross sections reflects that of the abundances of the light nuclei in nature. In a collision between a proton and an oxygen nucleus, the probability of production of ${ }^{11} \mathrm{~B}$ is higher than that of ${ }^{10} \mathrm{~B}$ which is itself higher than that of ${ }^{9} \mathrm{Be}$. Thus, it is not surprising that the abundances of these three isotopes go in declining order. This is a stricking example of a direct application of nuclear physics in the understanting of natural abundances. Note that the peaks of the cross sections lie at low energy especially that of alpha + alpha (Read \& Viola 1985). Thus low energy particles (about $10 \mathrm{MeV} / \mathrm{n}$ ) have to be inserted carefully in the treatment of the problem. Note also that at low energy, where the alpha + alpha reaction is operating at full strength, ${ }^{7} \mathrm{Li}$ and ${ }^{6} \mathrm{Li}$ are produced in comparable amounts (1.5), which is at variance with the ${ }^{7} \mathrm{Li} /{ }^{6} \mathrm{Li}$ ratio observed in meteorites (12.5). A stellar source of pure ${ }^{7} \mathrm{Li}$ has to be invoked to explain this high value (see above).

The production rate, in the most general case, is function i) of the number density of the target nuclei $\left(N_{T}\right)$ and their composition, ii) of the flux of the projectiles $(\Phi)$ and iii) of the cross section averaged over the energy spectrum in the interaction region :

\section{$\mathrm{dN}(\mathrm{L}) / \mathrm{dt}=N_{T}<\sigma>\Phi$.}

If $\mathrm{p}$ and alpha are the projectiles and CNO the targets, one deals with a "secondary" production, thus:

$\mathrm{dN}(\mathrm{L}) / \mathrm{dt}=N_{C N O}<\sigma>\Phi_{p, \alpha}$.

It is assumed (quite reasonably) that the flux of energetic particles is proportional to the supernova rate since they are thought to be the main agents of acceleration through the shock waves they produce. Due to the fact that SNII are also the main $\mathrm{O}$ producers, one expects that:

$\Phi(\mathrm{t})$ prop. $\mathrm{d}(\mathrm{SN}(\mathrm{t})) / \mathrm{dt}$ prop. $\mathrm{d}(\mathrm{O} / \mathrm{H}) / \mathrm{dt}$.

On the other hand, the CNO abundance cumulated in the ISM up to time $t$, is proportional to the total number of SN having exploded from 0 to $t$. Summarizing:

$\mathrm{d}(\mathrm{Be} / \mathrm{H}) / \mathrm{dt}$ prop. $(\mathrm{O} / \mathrm{H}) \mathrm{d}(\mathrm{O} / \mathrm{H}) / \mathrm{dt}$ or after integration $\mathrm{Be} / \mathrm{H}$ prop. $(O / H)^{2}$

In contrast, if $\mathrm{C}, \mathrm{O}$ are the projectiles and $\mathrm{H}$, He the targets, a primary production arises governed by the equation:

$\mathrm{dN}(\mathrm{L}) / \mathrm{dt}=N_{H H e}<\sigma>\Phi_{C O}$

Now with the same hypothesis than above, and considering that the target abundances $(\mathrm{H}, \mathrm{He})$ do not evolve significantly: 
$\mathrm{d}(\mathrm{Be} / \mathrm{H}) / \mathrm{dt}$ prop. $\mathrm{d}(\mathrm{O} / \mathrm{H}) / \mathrm{dt}$ and $\mathrm{Be} / \mathrm{H}$ prop. $\mathrm{O} / \mathrm{H}$

\section{Astrophysical agents and sites}

\subsection{Galactic Cosmic Rays (GCR)}

The energy spectrum of GCR is directly observed above, say, $1 \mathrm{GeV} / \mathrm{n}$. Below, it is deduced from various indirect observations (Strong \& Moskalenko 1999). It is reasonably well explained by the diffuse shock wave acceleration mechanism (Blandford \& Ostriker 1978; Jones \& Ellison 1991; Ellison et al 1997). The observed composition is extrapolated back to the sources thanks to a classical propagation model.

It is edifying to compare the elemental and isotopic source composition to that of other materials of known abundances (the solar system for instance, or that of the supernova ejecta computed with stellar models). Indeed SNIa alone do not fit $\mathrm{O} / \mathrm{Fe}, \mathrm{Ne} / \mathrm{Fe}, \mathrm{Mg} / \mathrm{Fe}$ neither the s process elements, whereas, SNII alone do not fit $\mathrm{Fe} / \mathrm{Co} / \mathrm{Ni}$, neither the s process elements. (see e.g. Meyer 1996, $2000)$. Indeed, the nucleosynthetic origin of the groups $(\mathrm{Mg}, \mathrm{Si}, \mathrm{Ca}, \mathrm{Fe}, \mathrm{Ni})$, ( $\mathrm{Sr}, \mathrm{Zr}, \mathrm{Ba}, \mathrm{Ce})$, ( $\mathrm{Pt}$ peak, actinides) are all different (explosive burning, sprocess and $r$ process, respectively) and their production sites are also different, thus the solar mix is a complex mixture of all that, historitically built up, and individual sources are unlikely to lead to the GCR source abundances (since they are similar to that of the solar system). Note that in this example we have chosen only refractory elements that are not supposed to be affected by selective effects (see below).

A stricking fact, of great importance for our purpose, is the similarity between the isotopic composition of the GCRs and of the solar system (Connell \& Simpson 1997; Stone et al 1998; Wiedenbeck et al 1999), which is a mixture of the products of generations and generations of stars of different masses indicating at face value that GCR are nuclei accelerated out of a normal reservoir and not strange, exotic, stars or objects.

In recent years, grains have been considered to play a central role in explaining the pecularities of the GCR source composition (Meyer et al 1997; Ramaty et al 1997). It is assumed that grain debris are more efficiently accelerated than elements in the gas phase and grain models have progressively replaced the traditional two step acceleration mechanism (injection by flare stars and acceleration by supernova shocks) in which grains were undesirable (Cassé \& Goret 1978, Meyer 1985; Silberberg \& Tsao 1990).

However the situation is not absolutely settled (Shapiro 1999, Silberberg et al 2000; Cassé \& Vangioni-Flam 2000, in preparation). Anyway, among the grain supporters themselves, there are divergent views concerning the origin and nature of the grains of interest. In one camp, they are supposed interstellar (Meyer and coworkers) and on the other they are supposed to contain fresh products of nucleosynthesis (Ramaty and colleagues). This has a strong bearing on the primary or secondary character of the LiBeB production mechanism. If the grains that are impacted by the accelerating shock waves have the ISM composition, then, the resulting CR composition should reflect that of the ISM which is the true reservoir of CR particles and, as it is $\mathrm{H}$, He dominated, the process is secondary. 
Ramaty and coworkers (see Ramaty et al 2000 and references therein) assume that GCR originate from grains loaded with freshly synthesized nuclei, $\mathrm{C}$ and $\mathrm{O}$ that are released by SNII and accelerated by shock waves in galactic superbubbles (SB). Subsequently, they interact with the surrounding interstellar medium to give $\mathrm{LiBeB}$ in a primary way. However, this proposal has to face the following objections: in the superbubble (SB), high temperature context, according to the observed trend (Cardelli 1996; Savage \& Sembach, 1996; Jenkins et al 1998; Howk, Savage \& Fabian 1999), grains have little chances to remain intact. Only their refractory cores would survive in the hot phase. Silicon, for instance would be significantly evaporated (see fig 5 in Savage \& Sambach 1996) with respect to $\mathrm{Fe}$, and the $\mathrm{Si} / \mathrm{Fe}$ ratio in the accelerated particles should be different than solar contrary to what is deduced from the GCR observations. Thus grains in SB are unlikely to account, in a detailed manner, for the present GCRS composition. Moreover, the ${ }^{34} \mathrm{~S} /{ }^{32} \mathrm{~S}$ ratio, derived at the cosmic ray sources poses also a problem (Cassé \& Vangioni-Flam 2000 in preparation). Finally, the grain mechanism does not go without gas $(\mathrm{H}, \mathrm{He})$ acceleration and thus particle accelerated in superbubbles cannot induce a purely primary process all time long and as the Galaxy evolves, the secondary process becomes more and more important. In this case, the transition primary-secondary will take place at about $[\mathrm{Fe} / \mathrm{H}]$ about -1 (taking an average superbubble metallicity of about 5 times solar, Higdon et al 1998). Thus a purely primary component seems unlikely.

On this basis we are tempted to conclude that standard GCR, as traditionally thought, act as a secondary source of $\mathrm{LiBeB}$, and if a primary component is made necessary by the data, it should be different from the standard GCR one. It could come from SB's under the condition that this SB component is confined to low energy not to spoil the observed CGR composition.

\subsection{Superbubble Accelerated Particles (SAP)}

Superbubbles gather a great number of massive stars which explode as type II supernovae, enriching the surrounding medium in fresh products of nucleosynthesis, and among them, oxygen. The shock wave and turbulence sutained by a given supernova, accelerate the material enriched by the previous ones (Bykov \& Fleisshman 1992; Bykov 1995; Bykov et al 2000). The energy spectrum obtained could depart significantly from the GCR one, depending on the detail of the mechamism and more precisely on the escape time of the fast particles, which is poorly known (Klepach et al 1999). As said previously, an energy cut off should be imposed to avoid contamination of the (observed) GCR abundances. This energy cut off is still a free parameter and we can choose it in order to avoid energetic problems (Ramaty et al 1996), i.e. in the range $30-300 \mathrm{MeV} / \mathrm{n}$. Then, all in all, superbubbles appear to be the best agents of a primary production process of LiBeB (Parizot \& Drury 1999; Bykov et al 2000). Admittedly, this theoretical proposal remains to be firmly (experimentally) substantiated by $\mathrm{X}$ ray and gamma-ray line observations (see the proceedings edited by Ramaty et al 1999 for a general discussion). The search for a non thermal low energy component as the one once claimed to be discovered by the COMPTEL experiment but discarded afterwards (Bloemen et al 1999) is, in our minds, one of the major objectives of the European INTEGRAL satellite to be lauched in 2001. For the 
time being, we can only speculate on the composition and the spectrum of this hypothetical (but somewhat necessary) component. In the halo phase, the composition is taken as representative of the ejecta of massive low metallicity stars. It is highly enriched in O w.r.t present CRs. (Woosley \& Weaver 1995). This composition however is expected to vary in time due to metallicity dependent mass loss rate (Vangioni-Flam et al 1997).

\section{Production and evolution of $\mathrm{LiBeB}$}

\subsection{Observed correlations}

Turning to observations, we are confronted to a certain ambiguity (as mentionned in the introduction). According to the choice of the conversion between $\mathrm{Fe}$ and $\mathrm{O}$ abundances we get different conclusions. Relying on the Israelian et al (1998) and Boesgaard et al (1999) relation we get Be proportional to $O^{1.7}$, i.e. nearly secondary, but taking the conventional $\mathrm{O}-\mathrm{Fe}$ relation, strengthened by the $\mathrm{Mg}$ data, we get $\mathrm{Be}$ proportional to $\mathrm{O}$, i.e. purely primary, at least in the halo phase. Thus the $\mathrm{O} / \mathrm{Fe}$ behaviour at low metallicity is a central issue, once again. Furthermore non LTE effects on B (Kiselman 1999) make the situation even more complex (see also Primas 2000). Thus we must rely on a independent argument to answer the question: is a primary component really necessary? This argument of energetic nature has been essentially developed by Ramaty et al $(1996,1997,2000)$. To briefly summarize: it seems that a primary component is required by both the $\mathrm{Mg}-\mathrm{Fe}$ relation (more secure than $\mathrm{O}-\mathrm{Fe}$ ) and by energetic arguments.

\subsection{The three evolutionary models}

Concerning the galactic evolutionary models, there exists three types of them i) a pure secondary standard GCR based on the variable $\mathrm{O} / \mathrm{Fe}$ ratio (Fields \& Olive 1999a) ii) a pure primary GCR from SB, which, suprisingly is still valid with flat or variable O/Fe (Ramaty et al 2000) iii) an hybrid model (Standard GCR + SAP based on a flat O/Fe ratio (Vangioni-Flam et al 1998) or based on a variable $\mathrm{O} / \mathrm{Fe}$ ratio (Fields et al 2000).

\subsection{Energetic requirements}

The number of atoms of Be produced per erg injected per supernova is promoted to the role of a selection criterion for the theoretical models (both primary and secondary). Stated differently, are there enough SNII and are they sufficiently efficient to produce all the Be observed in halo stars in the primary and/or secondary cases? According to Ramaty et al (2000) the pure secondary standard GCR produce $\mathrm{BeB}$ at high energy cost in the early Galaxy. SAP or primary GCR are much more economical. Thus a very plausible solution is that a primary component appears first, and a secondary component takes over afterwards, at a metallicity $(\mathrm{O} / \mathrm{H}$ ratio) which remains to be determined precisely. The position of the break depends on the Fe-O correlation used. Relying on the new one, and using the analysis based on the IRFM data, (Fields et al 2000) one finds a break point at about $[\mathrm{O} / \mathrm{H}]=-1.6$. On the other hand, if the ancient correlation is chosen, motivated by the magnesium data, the break point (if any) is at 
higher $[\mathrm{O} / \mathrm{H}]$ (about -1). Anyway, the existence of a primary component in the early stages of the evolution of the Galaxy seems mandatory, irrespective of the abundance data used, and this is a strong conclusion. Definitively, a primary component is required to fulfill the energetic constraint. What is the nature of this primary component? In our opinion, once again, it is distinct from GCR (assumed by Ramaty et al 2000 to originate from SBs), since, as said previously, we do not think that it is possible to identify SAP and GCR, as Higdon et al (1998) did, due to their different inferred composition.

Thus, we support the view that a low energy component is at work, complementing GCR to produce its lot of $\mathrm{LiBeB}$, specifically in the halo phase. Is this component still active? Nuclear gamma ray line astronomy will say. Thus our hopes are related to the INTEGRAL satellite. Our conclusion is that an hybrid model combining both a primary and a secondary components reconcile the abundance observations and energy requirement. The two components dominate sequentially. Of course the primary component, related to SAP would be overwhelming in the halo phase and is afterwards dominated by the secondary one, which constitutes the standard GCR. The SAP component plays a major role in the $\mathrm{LiBeB}$ production when the galactic gas is almost devoid of medium elements i.e. in the early Galaxy. Now, what is the ultimate reservoir of GCR? Grains in the ISM or stellar surfaces? This point is left to a future discusssion.

\section{Conclusion}

Ambiguities on abundance data preclude definitive conclusion: is $\mathrm{O} / \mathrm{Fe}$ flat or not at low Z? For the time being, the answer to this question depends on the observer to whom it is posed. Hopefully the debate will clarify in the next years. In the mean time we propose to rely on magnesium which is used as a secure metallicity index by a growing number of people. Special care should be taken to make NLTE corrections on Fe and B at low Z. A primary process probably is made necessary by energetic requirements. We endorse the view that two different components are responsible for the synthesis and evolution of $\mathrm{LiBeB}$ in the Galaxy. This hybrid model invoking the operation of both GCR (extracted by flares from stellar surfaces and/or grain debris in the ISM) subsequently accelerated by shock waves and a primary component of lower energy coming probably from superbubbles fulfil (or at least do not violate) all the composition constraints on i) the present cosmic radiation ii) the light element abundances in stars of all metallicities, including ${ }^{6} \mathrm{Li}$ (Vangioni-Flam et al 1999a; Fields \& Olive 1999b).

What to do next? Obviously measure. One would like idealy to get simultaneously the abundances of ${ }^{6,7} \mathrm{Li}, \mathrm{Be}, \mathrm{B}, \mathrm{O}, \mathrm{Mg}$ and $\mathrm{Fe}$ in the same star, this for many halo members of various metallicities, which does not seems out of reach of a dedicated VLT program (Cayrel, private communication). The solution of the $\mathrm{LiBeB}$ riddle is definitly in the hands of observers. The best way to reveal the presence of a low energy flux of $\mathrm{C}$ and $\mathrm{O}$ in the Galaxy related supperbubbles is the observation of broad gamma ray lines arising from their excitation and deexcitation in flight. The best hope to detect them in Vela, Orion and other star forming regions is offered by the european INTEGRAL satellite to be launched around 2001. 
Aknowledgements

We thank R. Cayrel and C. Furhmann for having drawn pure attention on the reliability of using magnesium as a metallicity index. We thank also Andrei Bykov and Vladimir Ptuskin for illuminating discussions on superbubbles.

\section{References}

Abia, C., Isern, J. \& Canal, R. 1993, A\&A, 275, 96

Balachandran, S. \& Bell, R.A. 1998, Nature, 392, 791

Barring, M. 2000, Summary-Rapporteur Volume of The 26th International Cosmic ray Conference, ed. B.L. Dingus (AIP, New-York). astro-ph/9912058

Blandford, R.D. \& Ostriker, J.P. 1978, ApJ, 221, L29

Bloemen, H. et al 1999, in "The Extreme Universe", 3rd INTEGRAL Workshop, Astrophysical Letters and Communications, vol. 38, 349

Boesgaard, A.M. \& King, J.R. 1993, AJ, 106, 2309

Boesgaard, A.M., King, J.R., Deliyannis, C.P. \& Vogt, S.S. 1999, AJ, 117, 492

Bykov, A.M. 1995, Space Sci. Rev., 74, 397

Bykov, A.M. \& Fleishman, G.D. 1992, MNRAS, 15, 269

Bykov, A.M., Gustov, M.Y. \& Petrenko, M.V. 2000, preprint

Cardelli, J.A. 1996, Science, 265, 209

Cassé, M. Lehoucq, R. \& Vangioni-Flam, E. 1995, Nature, 373, 38

Cassé, M. \& Goret, Ph. 1978, ApJ, 221, 703

Connel, J.J. \& Simpson, J.A. 1997, ApJ, 475, L61

Duncan, D., Lambert, D.L. \& Lemke, M. 1992, ApJ, 401, 584

Duncan, D. et al 1997, ApJ, 488, 338

Ellison, D., Drury, L.O'C. \& Meyer, J.P. 1997, ApJ, 487, 197

Fields, B.D. \& Olive, K.A. 1999a, ApJ, 516, 797

Fields, B.D. \& Olive, K.A. 1999b, New Astron., 4, 255

Fields, B.D., Olive, K.A., Vangioni-Flam, E. \& Cassé, M. 2000, astro-ph/9911320

Fuhrmann, K. 1998, A\&A, 338, 161

Fulbright , J.P. \& Kraft, R.P. 1999, AJ, 118, 527

Gilmore, J. et al 1992, Nature, 357, 379

Gustafsson, B. 1999, in "Chemical evolution from zero to high redshift", eds:

J.R. Walsh \& M.R. Rosa, Springer, 1

Hartmann, D., Myers, J. , Woosley, S., Hoffman, R. \& Haxton, H. 1999, in "LiBeB, Cosmic rays, and related X-and gamma-rays", edts: Ramaty et al. ASP Conf. Ser. vol 171, 235

Hernanz, M., José, J., Coc, A. \& Isern, J. 1996, ApJ, 465, L27

Higdon, B., Lingenfelter, R.E. \& Ramaty, R. 1998, ApJ, 309, L33

Howk, J.C., Savage, B.D. \& Fabian, D. 1999, ApJ, 525, 253

Israelian, G., Garcia-Lopez, R.J. \& Rebolo, R. 1998, ApJ, 507, 805

Jenkins, E.J. et al 1998, ApJ, 492, L147 
Jones, F.C. \& Ellison, D.C. 1991, Sp. Sci. Rev., 58, 259

Klepach, E.G., Psuthskin, V.S. \& Zirakashvili, V.N. 1999, Astroparticle Physics, in press

Kiselman, D. 1999, in"LiBeB Cosmic Rays, and Related X-Ray and Gammarays", Edts Ramaty et al, ASP Conf. Ser. vol 171, 85

McWilliam, A. 1997, ARA\&A, 35, 503

Meneguzzi, M., Audouze, J. \& Reeves, H. 1971, A\&A, 15, 337

Meyer, J.P. 1985, ApJS, 57, 173

Meyer, J.P. 1996, in "The Sun and beyond", 2nd Rencontre du Vietnam, Eds:

Tran Than Van et al, Edts frontières, 27

Meyer, J.P., Drury, L.O'C. \& Ellison, D.C. 1997, ApJ, 488, 730

Meyer, J.P. 2000, ISSI Workshop, Kluwer Academics Publishers, in press

Pagel, B.E.J \& Tautvaisiene, G. 1995, MNRAS, 276, 505

Pagel, B.E.J. 1999, astro-ph/9911204

Parizot, E. \& Drury, L.O'C. 1999, A\&A, 346, 339

Primas, F., Duncan, D.K., Peterson, R.C. \& Thorburn, J.A. 1999, A\&A, 313, 545

Primas, F. 2000, this conference

Ramaty, R., Kozlovsky, B. \& Lingenfelter, R.E. 1996, ApJ, 456, 525

Ramaty, R., Kovlowsky, B., Lingenfelter, R.E. \& Reeves, H. 1997, ApJ, 488, 730

Ramaty, R., Vangioni-Flam, E., Cassé, M. \& Olive, K.A. 1999, Proceedings

"LiBeB, Cosmic Rays, and Related X-and Gamma-Rays, ASP Conf. Ser., vol. 171

Ramaty, R., Scully, S., Lingenfelter, R.E. \& Kozlowsky, B. 2000, astro-ph/9909021, ApJ, in press

Read, S. \& Viola, R. 1985, Atomic Data Nucl. Data tables, 31, 359

Rebolo, R., Molaro, P. \& Beckman, J.E. 1988, A\&A 192, 192

Reetz, J. 2000, in "Galaxy Evolution: Connecting the distant Universe with Local Fossil Record", Eds M. Spite and N. Crifo, Astroph. Spc. Sci., in press

Reeves, H. 1994, Rev. Mod. Phys., 66, 193

Ryan, S.G., Norris, I., Bessel, M. \& Deliyannis, C. 1994, ApJ, 388, 184

Savage, B.D. \& Sembach, K.R. 1996, ARA\&A, 34, 279

Shapiro, M. 1999, in "LiBeB, Cosmic rays, and related X-and Gamma-Rays, Edts Ramaty el al, ASP Conf. Ser. vol. 171, 138

Shigeyama, T. \& Tsujimoto, T. 1998, ApJ, 507, L139

Silberberg, R. \& Tsao, C.H. 1990, ApJ, 352, L49

Silberberg, R. Tsao, C.H. \& Barghouty, A.F. 2000, in the 26th International Cosmic Ray Conference eds B.L. Dingus AIP (New York), OG.3.1.06, in press

Stone, E. et al 1998, Spc. Sci. Rev., 96, 285

Strong, A. \& Moskalenko, I. 1999, in "LiBeB, Cosmic rays, and related X-and Gamma-Rays, Edts Ramaty el al, ASP Conf. Ser. vol. 171, 162

Thomas, D., Greggio, L. \& Bender, R. 1998, MNRAS, 296, 119

Thielemann, F.K., Nomoto, K. \& Hashimoto, M. 1996, ApJ, 460, 408 
Umeda, H., Nomoto, K. \& Nakamura, W. 2000, to appear in the proceedings of the MPA/ESO conference "The first stars" eds A.Wess et al, Springer, astro$\mathrm{ph} / 9912248$

Vangioni-Flam, E., Cassé, M., Fields, B. \& Olive, K. 1996, ApJ, 468, 199

Vangioni-Flam, E., Cassé, M. \& Ramaty, R. 1997, in " The Transparent Universe", 2nd INTEGRAL Workshop, ESA, SP382, 123

Vangioni-Flam E., Ramaty, R., Olive, K.A. \& Cassé, M. 1998, A\&A, 337, 714

Vangioni-Flam, E. Cassé, M., Cayrel, R., Audouze, J., Spite, M. \& Spite F. 1999a, New Astronomy, vol. 4, no 4, 245

Vangioni-Flam, E., Ramaty, R., Cassé, M. \& Olive, K.A. 1999b, in "LiBeB, Cosmic-Ray and related X-and gamma-rays", Edts E. Ramaty et al, ASP Conf. Ser. vol. 171, 268

Vangioni-Flam, E. \& Cassé, M. 2000a, in "Galaxy Evolution: Connecting the distant Universe with the local fossil Record" Edts M. Spite, F. Crifo, Ap\&SS, in press

Vangioni-Flam, E. Cassé, \& Audouze, J. 2000b, Physics Report, in press

Webber, W.R., Kish, J.C. \& Schrier, D.A. 1990a, Phys. Rev. C 41, 520

Webber, W.R., Kish, J.C. \& Schrier, D.A. 1990b, Phys. Rev. C 41, 547

Wiedenbeck, M.E. et al 1999, ApJ, 523, L61

Woosley, S.E., \& Weaver, T.A. 1995, APJS, 101, 181 